\title{
Arrangement of Type IV Collagen and Laminin on Substrates with Controlled Density of $-\mathrm{OH}$ Groups
}

\author{
Nuno Miranda Coelho, (Ph.D. student) ${ }^{1,2}$ Cristina González-García, (Ph.D. student) ${ }^{3,4}$ \\ Manuel Salmerón-Sánchez, Ph.D., ${ }^{3,4}$ and George Altankov, M.D., Ph.D. ${ }^{1,3,5}$
}

Collagen IV (Col IV) and laminin (Lam) are the main structural components of the basement membrane where they form two overlapping polymeric networks. We studied the adsorption pattern of these proteins on five model surfaces with tailored density of $-\mathrm{OH}$ groups obtained by copolymerization of different ratios ethyl acrylate (EA) and hydroxyl EA (HEA): $\mathrm{X}_{\mathrm{OH}}=0, \mathrm{X}_{\mathrm{OH}}=0.3, \mathrm{X}_{\mathrm{OH}}=0.5, \mathrm{X}_{\mathrm{OH}}=0.7$, and $\mathrm{X}_{\mathrm{OH}}=1$ (where $\mathrm{X}$ refers the ratio of HEA). Atomic force microscopy revealed substratum-specific adsorption patterns of Col IV and Lam, ranging from single molecules deposition on more hydrophilic substrata to the formation of complex networks on hydrophobic ones. Human umbilical endothelial cells were used to study the biological performance of adsorbed proteins, following the overall cell morphology, the quantities for cell adhesion and spreading, and the development of focal adhesion complexes and actin cytoskeleton. Surprisingly, two optima in the cellular interaction were observed-one on the most hydrophilic $\mathrm{X}_{\mathrm{OH}}=1$ and other on the relatively hydrophobic $\mathrm{X}_{\mathrm{OH}}=0.3$ substrate-valid for both Col IV and Lam. When the proteins were adsorbed consecutively, a hydrophobic shift to $\mathrm{X}_{\mathrm{OH}}=0$ substratum was obtained. Collectively, these data suggest that varying with the density of $-\mathrm{OH}$ groups one can tailor the conformation and the functional activity of adsorbed basement membrane proteins.

\section{Introduction}

$\mathbf{T}$ ISSUE ENGINEERING STRIVES to replace the damaged tissue with scaffold (colonized or not with cells) that mimics the natural 3D organization of extracellular matrix (ECM).${ }^{1-3}$ However, the various currently used medical devices such as stents, prosthesis, and metal implants cannot avoid the 2D contact with tissues. Upon implantation they hamper the local organization of ECM and alter the biocompatibility of implant. The initial cellular events that take place at the biomaterials interface mimic to a certain extent their natural interaction with the ECM. ${ }^{4-6}$ Although the cells avoid direct contact with foreign materials, they readily attach to the adsorbed soluble matrix proteins such as fibronectin, vitronectin, fibrinogen, and others, ${ }^{3,5}$ which are uniformly available in the biological fluids. At longer contact with tissues, however, other less soluble ECM proteins such as collagens and Lam may also associate with the biomaterial surface influencing significantly the adjacent cells behavior and function., 3,8 Recently, we are particularly interested on the fate of adsorbed type IV collagen (Col IV) and Lam considering their important role for the assembly of basement membranes (BM) - a highly specialized ECM common to many types of tissues. ${ }^{9}$ The BM provides underlinement for the organ-specific cells supporting their functional organization. ${ }^{10}$ Consequently, the biohybrid organ technologies require construction of modules based on membranes in order to mimic the spatio-temporal organization and permeability of BM. ${ }^{3,11}$ However, despite the extensive studies on the role of BM components in various physiological and pathological conditions-ranging from the early development, wound healing, and angiogenesis, ${ }^{12}$ to the atherosclerosis, tissue fibrosis, and cancer ${ }^{13,14}$ _our knowledge on the behavior of Col IV and Lam at biomaterials interfaces is limited. Making use of atomic force microscopy (AFM) we recently described a distinct phenomenon of materialinduced assembly of Col IV on model hydrophilic and hydrophobic substrata. ${ }^{15}$ We found a spontaneous formation of a fine network on hydrophilic glass and a prominent polygonal network on hydrophobic octadecylsilane (ODS) that alters significantly Col IV activity. ${ }^{15}$ However, except on wettability the substrata we used differ in their chemistry,

\footnotetext{
${ }^{1}$ Institut de Bioenginyeria de Catalunya, Barcelona, Spain.

${ }^{2}$ Universitat Politècnica de Catalunya, Barcelona, Spain.

${ }^{3}$ CIBER de Bioingeniería, Biomateriales y Nanomedicina (CIBER-BBN), Valencia, Spain.

${ }^{4}$ Center for Biomaterials, Universidad Politécnica de Valencia, Valencia, Spain.

${ }^{5}$ ICREA (Institució Catalana de Recerca i Estudis Avançats), Catalonia, Spain.
} 
which rise obstacles for interpretation of results. Moreover, we did not consider the behavior of the other main BM player-the Lam-particularly in conditions where both proteins are adsorbed. Therefore, here we describe the materialdriven arrangements of adsorbed Col IV and Lam on model surfaces ${ }^{16}$ where fraction of $-\mathrm{OH}$ groups vary as an independent parameter. Using this well-defined family of polymer substrata, ${ }^{16}$ we recently described the adsorption pattern of various ECM proteins, including fibronectin, ${ }^{17}$ vitronectin, ${ }^{18}$ and fibrinogen, ${ }^{19}$ showing that it may strongly influence their biological activity. ${ }^{16-18}$ Here we extend these studies corroborating the substrate behavior Col IV and Lam as main BM constituents. To follow their biological performance we choose primary endothelial cells considering their role for the functional organization of vascular BM and their involvement in the neovascularization ${ }^{3,11}$ both events critically important for most tissue engineering applications. ${ }^{11,12}$

Here we demonstrate that the adsorption patterns of both Col IV and Lam are strongly dependent on the surface fraction of $-\mathrm{OH}$ groups. AFM studies revealed specific substratum-induced assembly of these proteins, from single molecule arrangements to the specific networks-the later better pronounced on hydrophobic environment. The initial interaction of endothelial cells surprisingly shows two optima-a hydrophilic $\left(\mathrm{X}_{\mathrm{OH}}=1\right)$ and a hydrophobic one $\left(\mathrm{X}_{\mathrm{OH}}=0.3\right)$ - evaluated from the changes in adhering cells morphology, the quantities for cell adhesion and spreading, and the development of focal adhesions and actin cytoskeleton. When both proteins were adsorbed consecutively, a distinct complex morphology of the adsorbed protein layer was observed resulting in a hydrophobic shift in the cellular interaction (to $\mathrm{X}_{\mathrm{OH}}=0$ ). Details of this study are reported herein.

\section{Materials and Methods}

\section{Materials}

Copolymer sheets were obtained by polymerization of a solution of both monomers ethyl acrylate (EA) (Aldrich, 99\% pure) and hydroxyl EA (HEA) (Aldrich, 96\% pure), in the desired proportion, using $0.1 \mathrm{wt} \%$ of benzoin (Scharlau, $98 \%$ pure) as photoinitiator and a $2 \mathrm{wt} \%$ of ethyleneglycol dimethacrylate (Aldrich, $98 \%$ pure) as crosslinking agent. The polymerization was carried out up to limiting conversion and against a smooth glass surface seeking to obtain surfaces of controlled and reproducible roughness. Five monomer feed compositions were chosen, given by the weight fraction of HEA in the initial mixture of $\mathrm{X}_{\mathrm{OH}}=1,0.7,0.5,0.3$, and 0 (it refers to the fraction $X$ of HEA in the copolymer). After polymerization, low molecular mass substances were extracted from the material by boiling in ethanol for $24 \mathrm{~h}$ and then drying in vacuo to constant weight.

The equilibrium water content (mass of water absorbed referred to the dry mass of the substrate) and the water contact angle (using a Dataphysics OCA) were measured for the different substrates.

Small disks ( $\sim 10 \mathrm{~mm}$ diameter) were cut from the polymerized sheets to be used in the protein adsorption and cell adhesion studies. The samples were sterilized with gamma radiation $(25 \mathrm{kGy})$ before the experiments.

XPS experiments were performed in a PHI 5500 Multitechnique System (from Physical Electronics) with a mono- chromatic X-ray source and calibrated using the $3 \mathrm{~d}_{5 / 2}$ line of Ag. The analysed area was a circle of $0.8 \mathrm{~mm}$ diameter, and the selected resolution for the spectra was $23.5 \mathrm{eV}$ of pass energy and $0.1 \mathrm{eV} \cdot \mathrm{step}^{-1}$. All measurements were made in an ultra high vacuum chamber pressure. XPS elemental sensitivity factors according to the MULTIPAK program for PHI instruments were used. An automatic XPS signal fitting software has been developed under MATLAB v7.2 (The MathWorks, Inc.) environment to deconvolute the experimental spectra as described elsewhere. ${ }^{20}$ The fitting software makes use of an independent Voigt function per peak (which in our case means four Voigt functions to fit the $C$ 1s spectra and three Voigt functions to fit the $\mathrm{O} 1 \mathrm{~s}$ peaks).

\section{Protein adsorption}

The adsorption concentration for both proteins was $20 \mu \mathrm{g} /$ $\mathrm{mL}$ : in $0.1 \mathrm{M}$ sodium acetate ( $\mathrm{pH} 4.5$ ) for Col IV (Abcam) and in phosphate-buffered saline (PBS) ( $\mathrm{pH}$ 7.4) for LAM (Sigma). Some samples were coated consecutively, first with Col IV than Lam, with extensive washing with PBS between incubations. The incubation period was of $10 \mathrm{~min}$ for AFM studies and $30 \mathrm{~min}$ for cellular interaction, both at $37^{\circ} \mathrm{C}$. Afterward, the samples were extensively washed with PBS.

\section{AFM}

Samples with the adsorbed protein were quickly dried using Nitrogen air flow. AFM was performed in a NanoScope III from Digital Instruments operating in tapping mode in air. Si-cantilevers from Veeco were used with force constant of $2.8 \mathrm{~N} / \mathrm{m}$ and resonance frequency of $75 \mathrm{kHz}$. The phase signal was set to zero at a frequency $5 \%-10 \%$ lower than the resonance one. Drive amplitude was $200 \mathrm{mV}$ and the amplitude set-point $\mathrm{A}_{\mathrm{sp}}$ was $1.4 \mathrm{~V}$. The ratio between the amplitude set-point and the free amplitude $\mathrm{A}_{\mathrm{sp}} / \mathrm{A}_{0}$ was kept equal to 0.7. The WSxM software was used to process height AFM images to obtain topographical profile (number of event per given altitude) and a tailor-made MATLAB software (The MathWorks, Inc.) for volume calculations of the adsorbed protein layer. Volume protein calculated following this procedure must be interpreted in a relative way. That is to say, the total real volume of the adsorbed protein must be higher due to the hydration effects. Moreover, our calculated protein volume cannot be directly used to infer protein mass density on each surface. Nevertheless, assuming the drying process will equally modify both Col IV and Lam on every surface, the obtained values provide valuable information to compare the amount of adsorbed protein among surfaces and the influence of their sequential adsorption.

\section{Cells}

Human umbilical vein endothelial cells (HUVEC, PromoCell) were cultured in endothelial cell growth medium supplemented with SupplementMix (PromoCell) containing $0.4 \%$ ECGS $/ \mathrm{H}, 2 \%$ fetal calf serum, $1 \mathrm{ng} / \mathrm{mL}$ epidermal growth factor, $1 \mu \mathrm{g} / \mathrm{mL}$ hydrocortison, and $1 \mathrm{ng} / \mathrm{mL}$ basic fibroblast factor. For the adhesion experiments the cells were detached from around confluent flasks with Trypsin/EDTA (Invitrogen) and the remained trypsin activity was stopped with 100\% FBS before two times washing with medium without supplements. Finally, the cells were counted and 
reconstituted in serum-free endothelial cell medium to be seeded on the different surfaces.

\section{Overall cell morphology}

To study the overall cell morphology of HUVEC adhering to different samples, $5 \times 10^{4}$ cells / well were seeded in 24-well TC plates (Costar) containing the samples for $2 \mathrm{~h}$ in the serum-free medium. At the end of incubation, the cells were fixed with $4 \%$ paraformaldehyde $(10 \mathrm{~min})$ and permeabilized with $0.5 \%$ Triton X-100 for $5 \mathrm{~min}$. For the overall morphology studies and cell adhesion measurements the actin cytoskeleton was stained with $20 \mu \mathrm{g} / \mathrm{mL}$ AlexaFluor 488 phalloidin (Molecular Probes) in PBS before the samples were washed and mounted in Mowiol. In some cases phalloidin was added to the secondary antibody solution (for double vinculin/ actin staining). The samples were viewed on fluorescent microscope (Nikon Eclipse E800) and at least three representative images were acquired.

\section{Quantification of cell adhesion and spreading}

Morphological parameters such as number of adhering cells and mean cell surface area were evaluated using the ImageJ plug-ins (NIH; http://rsb.info.nih.gov/ij/). The adhesion was measured by counting the cells in three randomly chosen images of actin-stained samples to obtain the number of cells per $\mathrm{cm}^{2}$. Data were collected from at least three independent experiments and the average cell area was further measured for each individual image (in $\mu^{2}$ ) and calculated for each condition.

\section{Immunofluorescence}

Observation of focal adhesion contacts. About $5 \times 10^{4}$ cells/well were seeded as above for $2 \mathrm{~h}$ in serum-free medium. To observe focal adhesions, fixed and permeabilized samples were saturated with 1\% albumin in PBS for $15 \mathrm{~min}$. Focal adhesion complexes were observed using monoclonal anti-vinculin antibody (Sigma) dissolved at $1 \%$ albumin solution in PBS for 30 min followed by $\mathrm{Cy}^{3}$-conjugated AffiniPure Goat Anti-Mouse IgG $(\mathrm{H}+\mathrm{L})$ (Jackson ImmunoResearch) as secondary antibody. The samples were viewed and photographed on a Laser Scanning Confocal Microscope Leica at high magnification where at least three representative images were acquired for each experimental condition.

Statistical analysis. All cellular studies were performed with at least three replicates. Morphological quantification for each surface and each condition was obtained from at least six representative pictures, which were digitally analyzed. Statistics was performed using StatGraphics Plus software employing ANOVA simple and Kruskal-Wallis tests to determine statistically significant differences between groups $(p<0.05)$. Each data point represents mean \pm standard deviation from at least three independent experiments.

\section{Results and Discussion}

Col IV and Lam are the main component of the vascular $\mathrm{BM}^{11,12}$ where endothelial cells reside in a rather $2 \mathrm{D}$ environment, for example, in a similar geometry when adhering to the surface of blood contacting devices where the endothelization is highly desirable., ${ }^{71,12}$ However, while en- dothelial cells procurement technologies for seeding implants have significantly improve, adhering cells often dedifferentiate and act in a counterproductive manner, accelerating device failure. ${ }^{3,4,6} \mathrm{We}$ anticipate that an important factor for this altered endothelization response might be the missing environmental signals from the natural BM. Thus, tailoring the behavior of adsorbed Col IV and Lam on biomaterials interface would provide a powerful tool (from the materials site) for maintaining the functional performance of implant.

It is well documented that the substratum $-\mathrm{OH}$ groups strongly affect both the conformation and biological performance of adsorbed proteins. ${ }^{17,19,21,22}$ Relatively little is known, however, about the intermolecular associations of adsorbed protein molecules, particularly when they tend to assembly within the protein layer. In this study we used a family of model substrates based on the copolymerization of EA and HEA monomers, which have a vinyl backbone chain with the side groups $-\mathrm{COOCH}_{2} \mathrm{CH}_{3}$ and $-\mathrm{COOCH}_{2} \mathrm{CH}_{2} \mathrm{OH}$, respectively. Their combination gives rise to a random copolymer $^{22}$ with tailored concentration of $-\mathrm{OH}$ groups that determines both the surface energy and the hydrophilicity of the substrate (Table 1).

Topography of the surfaces examined by AFM prior protein adsorption yielded similar roughness parameters regardless the polymer composition. Roughness parameters obtained were $R a=18 \pm 4 \mathrm{~nm}$ and $R m s=22 \pm 4 \mathrm{~nm}$ ( $R a$ is the arithmetic average of the height deviations; Rms is defined as standard deviation of the height values).

The fraction of $-\mathrm{OH}$ groups on the surface was assessed by XPS. Table 1 includes the surface HEA molar fraction for each of the copolymers. It is noteworthy that the fraction of hydrophilic units on the surface is similar to the initial ratio of HEA in the feeding mixture of co-monomers. That is to say, most of the hydroxyl groups in the system remain available to interact with the biological media.

\section{Behavior of adsorbed Col IV on surfaces with different $-\mathrm{OH}$ content}

The network-forming Col IV is a triple helical molecule composed of three alpha chains, which self-assemble by antiparallel interactions and extensive disulfide bounding. ${ }^{10}$ In the $\mathrm{BM}$ these monomers associate with their C-terminal globular NC1 domains to form dimers or with N-terminals to form tetramers. ${ }^{10,23,24}$ Observations have been made that

\begin{tabular}{|c|c|c|c|}
\hline $\mathrm{OH}$ & EWC (\%) & $W C A\left(^{\circ}\right)$ & $X_{\mathrm{OH}}(\mathrm{XPS})$ \\
\hline & $1.7 \pm 0.4$ & $89 \pm 1$ & 0 \\
\hline 3 & $7.6 \pm 0.9$ & $80 \pm 2$ & $0.25 \pm 0.05$ \\
\hline 5 & $18.2 \pm 1.7$ & $67 \pm 1$ & $0.45 \pm 0.08$ \\
\hline .7 & $40.6 \pm 0.4$ & $55 \pm 1$ & $0.68 \pm 0.06$ \\
\hline & $134 \pm 5$ & $45 \pm 2$ & 1 \\
\hline
\end{tabular}

The first column represents the fraction of HEA units used in the copolymerization process; the last one is the fraction of HEA units available on the material surface as obtained from XPS analysis.

EWC, equilibrium water content; WCA, water contact angle; HEA, hydroxylethyl acrylate. 
Col IV could be linearly organized during early BM assembly, ${ }^{25}$ suggesting that cells are also involved in the process. While the molecular mechanisms that endow the spatial organization of Col IV in the BM are still debatable, our current results $^{15,26}$ suggest that material surface-associated Col IV may undergo cell-dependent rearrangement, presumably through reversible association with FN fibrils - a fact that brings forth our interest on the molecular arrangement of BM proteins at biomaterials interface.

Structure of Col IV molecule was extensively studied during last decades ${ }^{23,24,27}$ and AFM provide significantly insight. ${ }^{28}$ AFM is an exceptional tool to explore the conformation and distribution of matrix proteins at the biomaterial interface. Since roughness for most of the biomaterials surfaces is well above the height of protein molecules $(5-10 \mathrm{~nm})^{28}$ direct observations of ECM proteins deposition on commonly used biomaterials are sparsely reported. In previous research we could show that the phase magnitude in tapping mode AFM is a good approach to obtain significant information on protein configuration. $^{21}$ The reason why we have chosen working in air in this study is mainly due to the stability of the system, which continues to be much better in air compared to the scanning in liquid environments. For example, we have found that on hydrophobic materials, images obtained in air are comparable to those acquired in liquid but with much better resolution. By contrast, on very hydrophilic surfaces that moreover adsorbed large amounts of water, the interpretation of the AFM images must be done carefully.

The AFM phase images shown on Figure 1A describe the surface behavior of adsorbed Col IV depending on the fraction of $-\mathrm{OH}$ groups, which vary from $\mathrm{X}_{\mathrm{OH}}=0$ to $\mathrm{X}_{\mathrm{OH}}=1$. Two general trends might be distinguished: (i) single molecules arrangement (see arrowhead on $\mathrm{X}_{\mathrm{OH}}=0.5$ ) characteristic for the intermediate hydrophobic substrata, and (ii) a tendency for molecular assembly in network (see arrow on $\mathrm{X}_{\mathrm{OH}}=0.7$ ), which increase with hydrophobicity, leading to the formation of an augmented network structure at $\mathrm{X}_{\mathrm{OH}}=0$, presumably consisting of molecular aggregates. Elongated fibril-like features were observed also on the most hydrophilic poly EA; equal to $\mathrm{X}_{\mathrm{OH}}=0$ (PEA) surface presumably representing few laterally associated molecules (arrowhead on $\mathrm{X}_{\mathrm{OH}}=1$ ). When Col IV adsorbs on mica it typically forms dimers through intermolecular interactions of two Col IV monomers via the globular NC1 domains. ${ }^{28}$ Indeed, such globular features connected with long arms can be easy distinguished on intermediate hydrophilic $\mathrm{X}_{\mathrm{OH}}=0.5$ and $\mathrm{X}_{\mathrm{OH}}=0.3$ samples (see arrowhead on $\mathrm{X}_{\mathrm{OH}}=0.5$ ). Even on mica, however, Col IV dimers display a variety of configurations often forming loops and folds, ${ }^{28}$ which may explain the appearance of rather complex shapes seen on $\mathrm{X}_{\mathrm{OH}}=0.7$. The formation of tetramers via interactions of $7 \mathrm{~S}$ domains is also feasible ${ }^{28}$ (see arrow on $\mathrm{X}_{\mathrm{OH}}=0.3$ ) presumably involved in the formation of networks (upon decrease of -OH content) as single tetramer features were not observed. Nevertheless, the longitudinal sizes of obtained structures vary between 300 and $600 \mathrm{~nm}$ (see arrowhead on $\mathrm{X}_{\mathrm{OH}}=0.5$ ), which is in the range of individual molecules size of unfolded Col IV monomer $(346+3.8 \mathrm{~nm})^{28}$ and dimers might be maximally doubled, for example, around $700 \mathrm{~nm}$, but when are completely unfolded. The Col IV arrangement on $\mathrm{X}_{\mathrm{OH}}=0.3$ is more subtle. Here the links between individual molecules cause a tendency for network assembly. Since there are no
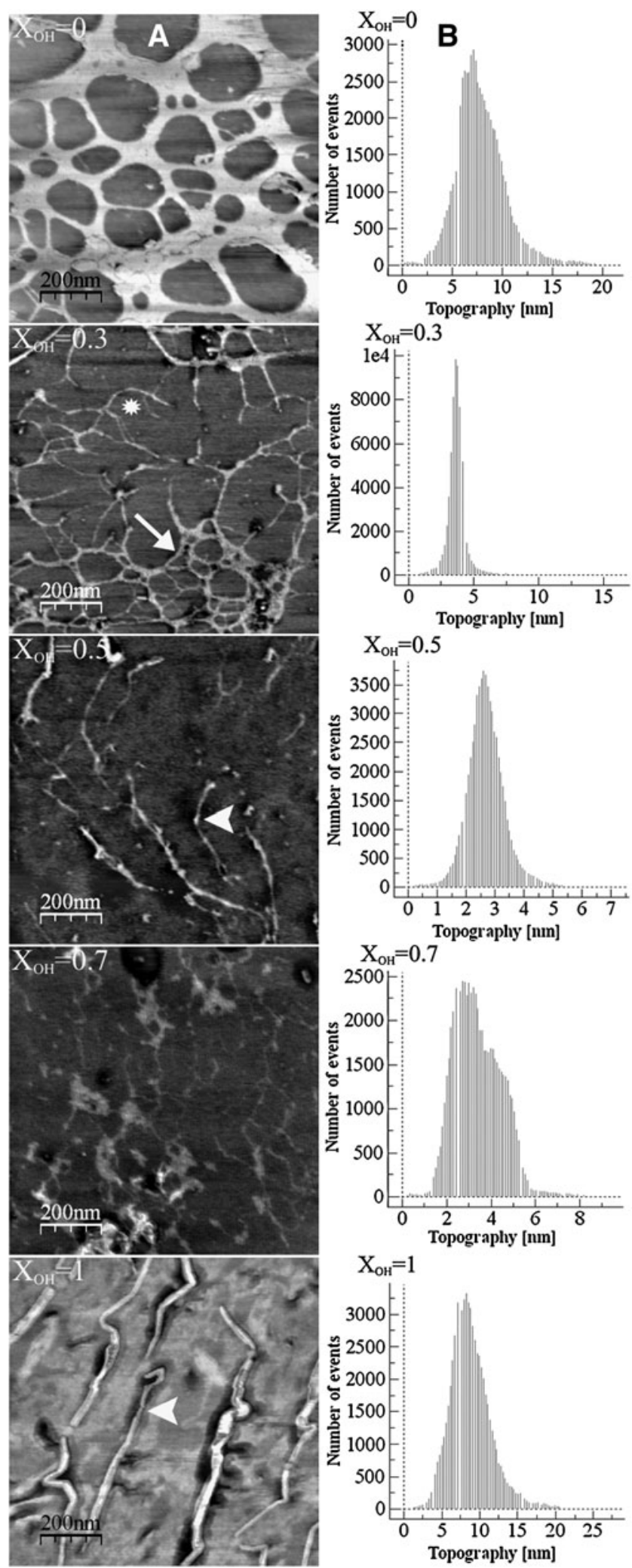

FIG. 1. Phase AFM images of adsorbed Col IV on substrates with increasing fraction of $-\mathrm{OH}$ groups (A) following the order: $\mathrm{X}_{\mathrm{OH}}=0, \mathrm{X}_{\mathrm{OH}}=0.3, \mathrm{X}_{\mathrm{OH}}=0.5, \mathrm{X}_{\mathrm{OH}}=0.7, \mathrm{X}_{\mathrm{OH}}=1$ from the top to the bottom. Corresponding topographic profiles of adsorbed Col IV on the same substrates (B) viewed by AFM software (see Methods section). AFM, atomic force microscopy; Col IV, collagen IV. 
overlapping regions, arrangement at single molecules level is very probable. More precise topographical analysis also shows the most homogenous size distribution of the features on this sample (Fig. 1, column B) suggesting formation of complex structure consisting of small repeating units. That is to say, the topography histograms (1B) show a single distribution of molecules within the interval $5-15 \mathrm{~nm}$ for most of the samples that even narrows to $2-6 \mathrm{~nm}$ for $\mathrm{X}_{\mathrm{OH}}=0.3$. Indeed, we cannot discard that the formation of structures on this surface is partly a consequence of the drying process, which could lead to lateral reorganization of the adsorbed layer at the air-liquid interface, but this process seems to be favored on very hydrophilic surfaces because of the absence of protein-surface interactions strong enough to prevent protein relaxation during water release. ${ }^{39}$

As shown in Table 2, the total amount of adsorbed Col IV decreases sharply as the hydrophilicity of the sample increases and then remains in barely constant. Conversely, on pure PEA $\left(\mathrm{X}_{\mathrm{OH}}=0\right)$ the process turns to the formation of an augmented network that enhances interactions between molecules, resulting in the formation of a complex protein structure (aggregate) where individual molecules cannot be distinguished any more. This arrangement of Col IV on PEA $\left(\mathrm{X}_{\mathrm{OH}}=0\right)$ occurs as on hydrophobic ODS but with different volumetric distribution: multilayer aggregates on ODS versus lateral interactions on $\mathrm{X}_{\mathrm{OH}}=0 .{ }^{15}$ This fact supports the idea that the concrete underlying chemistry-not only the wettability of the substrate-determines protein adsorption and distribution at the material interface. ${ }^{16,17}$

\section{Biological response to the adsorbed Col IV}

The surface wettability has long been recognized as an important parameter for protein adsorption and cellular interaction. 5,16,29 In general, hydrophilic surfaces support cell adhesion and spreading, usually attributed to the appropriate conformation of adsorbed matrix proteins. ${ }^{5,21,30,31}$ However, this is not always straightforward since materials with very high wettability, which bind much water-like hydrogels, do not support protein adsorption and cell adhesion. ${ }^{32}$ Our study shows a very good interaction of HUVEC with the most hydrophilic $\mathrm{X}_{\mathrm{OH}}=1$ substratum, which is not surprising, as this surface might be considered as moderately wettable where the best cellular interaction is expected. ${ }^{32,33}$ Indeed, the cells look well spread (Fig. 2A, left column) and develop an elongated morphology. The promoted cellular interaction is confirmed by the quantitative measurements for the adhesion (Fig. 2D, left chart) and spreading (Fig. 2D,

Table 2. Total Volume Occupied by the Protein on a $1 \mu \mathrm{M}^{2}$ Area of the Substrate as Calculated from AFM Data After Adsorption of Col IV, LAM, OR COL IV + LAM

\begin{tabular}{lccc}
\hline$X_{\mathrm{OH}}$ & Col IV & Lam & Col IV/Lam \\
\hline 0 & $6.0 \pm 1.1$ & $5.0 \pm 1.2$ & $8.0 \pm 1.3$ \\
0.3 & $2.1 \pm 0.3$ & $3.0 \pm 0.9$ & $3.5 \pm 1.1$ \\
0.5 & $0.7 \pm 0.2$ & $2.5 \pm 0.8$ & $3.7 \pm 1.2$ \\
0.7 & $1.5 \pm 0.2$ & $0.7 \pm 0.2$ & $6.0 \pm 1.4$ \\
1 & $1.2 \pm 0.3$ & $6.0 \pm 1.5$ & $4.5 \pm 1.3$ \\
\hline
\end{tabular}

AFM, atomic force microscopy; Col IV, Collagen IV; Lam, Laminin. right), both optima showing a significant increase over other conditions $(p<0.05)$ Surprisingly, however, HUVEC represents a second optimum for cell interaction, that is on the relatively hydrophobic $\mathrm{X}_{\mathrm{OH}}=0.3$ surface (hydrophobic effect), where adhesion and spreading again significantly $(p<0.05)$ improve (Fig. 2A). For comparison, on the same chart are shown the quantities for adhesion and spreading to glass (as a positive control). It can be seen that adhesion, but not spreading, of cell on $\mathrm{X}_{\mathrm{OH}}=0.3$ is significantly higher, whereas on $\mathrm{X}_{\mathrm{OH}}=1$ it equal with the control. The spreading area is equal for all $\mathrm{X}_{\mathrm{OH}}=0.3, \mathrm{X}_{\mathrm{OH}}=1$, and control samples. The overall morphology of cells on the control sample might be seen on Fig. 5C. Further decrease of $-\mathrm{OH}$ groups $\left(\mathrm{X}_{\mathrm{OH}}=0\right.$ surface) tends to diminish the cellular interaction. HUVEC fail to interact also with the intermediate fractions of $\mathrm{X}_{\mathrm{OH}}=$ 0.5 and $\mathrm{X}_{\mathrm{OH}}=0.7$ : overall, the cells tend to round (Fig. 2, row A) and both adhesion and spreading show significantly lower $(p<0.05)$ quantities (Fig. 2D), all this confirming the optimum at $\mathrm{X}_{\mathrm{OH}}=0.3$. Nevertheless, on the $\mathrm{X}_{\mathrm{OH}}=0$ and partly $X_{\mathrm{OH}}=0.3$ surfaces, the cells represent an irregular shape with often appearance of star-like protrusions, rich on actin (see Fig. 2A and C), but normal focal contacts formation (Fig. 2B), which suggest a rather good cellular interaction. To rationalize this cellular behavior in terms of protein adsorption shown on Figure 1, it suggests that supramolecularsized fibrillar features formed on the $\mathrm{X}_{\mathrm{OH}}=1$ substrate favor cell adhesion; that is to say, Col IV is adsorbed in such a way that it provides enhanced availability of the binding site (for $\alpha 1$ and $\alpha 2$ integrins) located $\sim 100 \mathrm{~nm}$ away from the aminoterminus. ${ }^{23,24,27}$ As the hydrophilicity of the substrate diminishes, protein distribution became complicated, from more complex features seen on $\mathrm{X}_{\mathrm{OH}}=0.7$ (see Fig. 1A), the isolated fibrils are more characteristic for $\mathrm{X}_{\mathrm{OH}}=0.5$ (Fig. 1A), but why these structures appear worse for the cellular interaction is not clear. Moreover, lateral protein interactions are enhanced on $\mathrm{X}_{\mathrm{OH}}=0$, which must hidden binding domains resulting in poorer cell interaction.

\section{Assembly of LAM on surfaces with different $-\mathrm{OH}$ density}

It is proposed that the assembly of BM is initiated by Lam. It self-assembles into heterotrimers that bind to the cell surface via integrin receptors. ${ }^{34-36}$ The structure of Lam molecules has been extensively studied during the recent decades employing electron microscopy and also AFM. ${ }^{16,37,38}$ Consistent with this studies an appearance of cross-shaped molecules with approximate size of $70-100 \mathrm{~nm}$ (depending on their conformation) might be expected as the maximal dimensions of the completely extended cruciform Lam molecule is $125 \mathrm{~nm}$ long, $72 \mathrm{~nm}$ wide, and $2.2 \mathrm{~nm}$ thick. ${ }^{37}$

The phase images on Figure 3A show Lam distribution after adsorption from solution of a high concentration $(20 \mu \mathrm{g} / \mathrm{mL})$. Note, although such concentration is not optimal for AFM imaging, it is the one used for the cellular investigations. We found a clear tendency for protein assembly in networks at almost all ranges of surfaces (Fig. 3, column A). However, these networks vary significantly in their thickness and organization: on substrates with low $\mathrm{OH}$ content, these structures are prominent $\left(\mathrm{X}_{\mathrm{OH}}=0-\mathrm{X}_{\mathrm{OH}}=0.5\right)$, whereas a rather subtle protein deposition is characteristic for $\mathrm{X}_{\mathrm{OH}}=1$ and $\mathrm{X}_{\mathrm{OH}}=0.3$ surfaces (see Fig. 3, row A). It 
FIG. 2. Overall morphology of HUVEC adhering on Col IV-coated substrates with increasing $-\mathrm{OH}$ density (A). Bar $100 \mu \mathrm{m}$. The same samples were stained for vinculin (B) and actin (C) to study the formation of focal adhesion contacts and actin cytoskeleton organization, respectively. Bar $10 \mu \mathrm{m}$. The quantities for cell adhesion expressed as number of cells per $\mathrm{cm}^{2}$ (D, left graph) and cell spreading area shown in $\mu \mathrm{m}^{2}$ (D, right graph) are compared to the control glass samples (white bars). HUVEC, human umbilical vein endothelial cells.
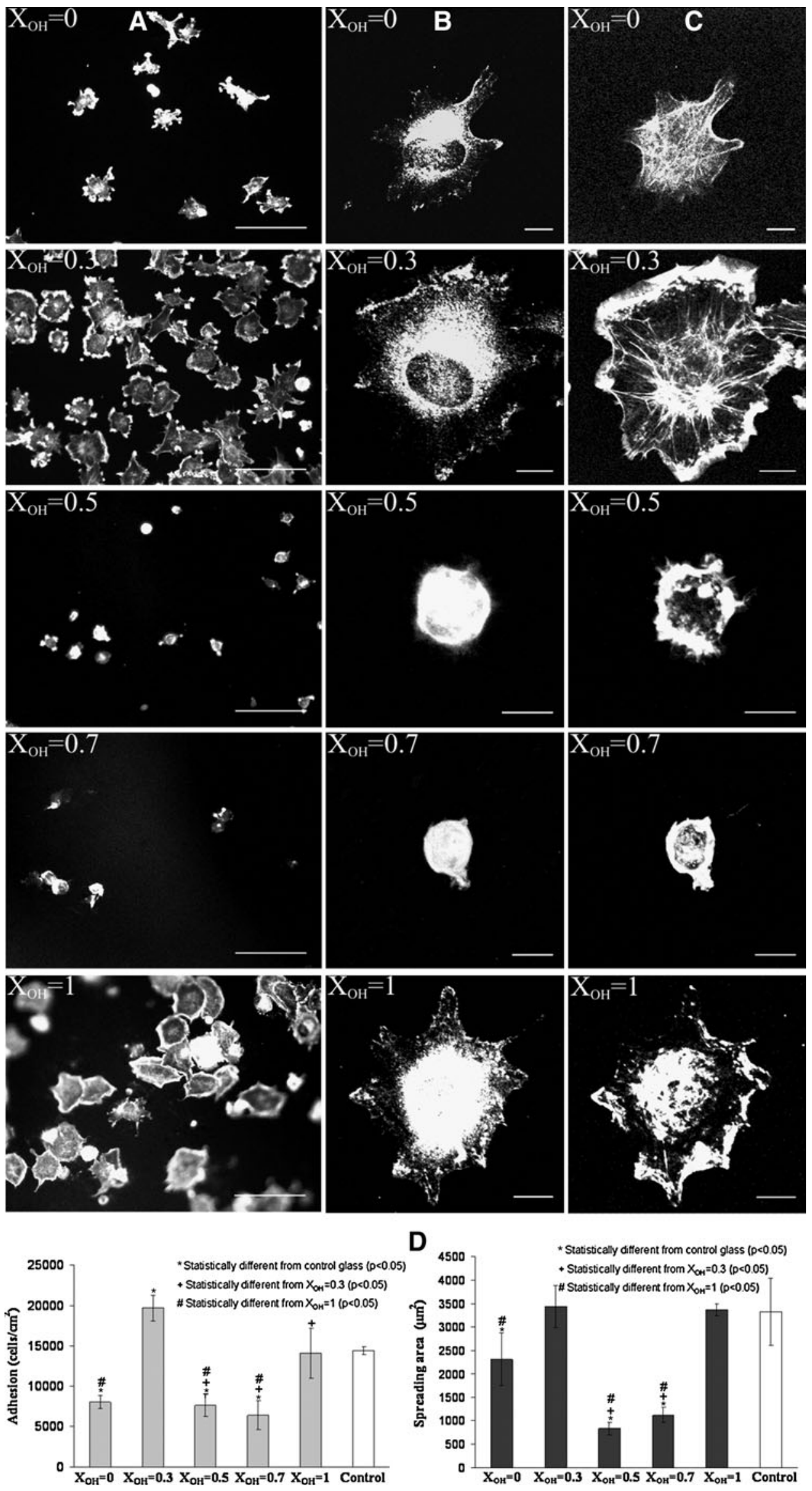

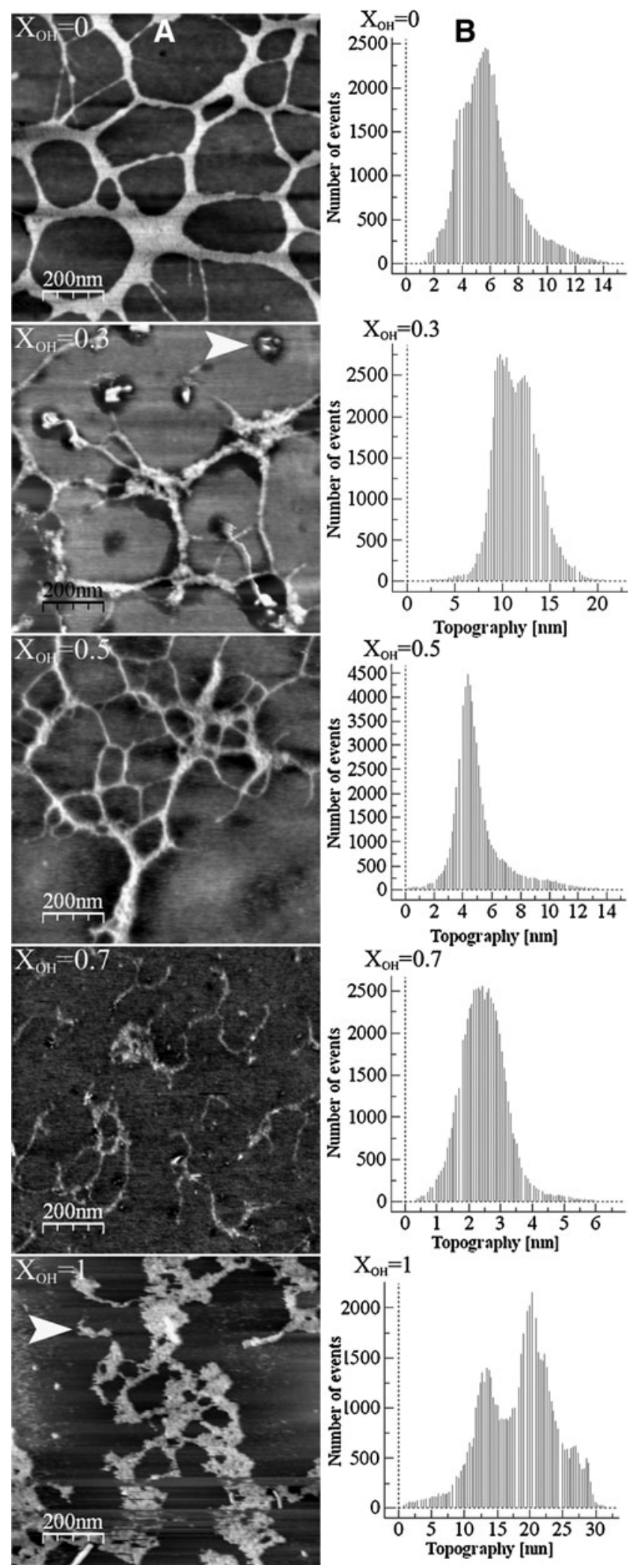

FIG. 3. Phase AFM images of adsorbed Lam on substrates with increasing fraction of $-\mathrm{OH}$ groups (A) following the same order as in Figure 1. Corresponding topographic profiles of adsorbed Lam on the same substrates (B) viewed by AFM software. Lam, Laminin. seems that increasing the fraction of $-\mathrm{OH}$ groups tend to diminish the network formation presumably reducing the lateral intermolecular interactions. However, on most hydrophilic $\mathrm{X}_{\mathrm{OH}}=1$ surface, Lam shows again surprisingly high protein deposition as extrapolated from the calculations for the adsorbed protein volume (Table 2). The same nonmonotonic trend in the amount of adsorbed protein was previously found on this family of surfaces using fibronectin. ${ }^{21}$ Note, on the same surface we have previously found globular-like Lam deposition after adsorption from solutions of much lower protein concentration, ${ }^{16}$ which stress the importance of the competition between protein-protein versus protein-material interactions to determine the conformation of LAM at the material interface. On a collateral side, swelling of polymer may result in the entrapment of some Lam aggregates within the polymer, which may also explain the biphasic size distribution of features seen on $\mathrm{X}_{\mathrm{OH}}=1$ substratum only (Fig. 3, column B, bottom). Also, the drying process could lead to lateral reorganization of the adsorbed protein layer at the air-liquid interface, and this process seems to be favored on very hydrophilic surfaces because of the absence of protein-surface interactions strong enough to prevent protein relaxation during water release. ${ }^{39}$ Nevertheless, independently of the complex arrangements of Lam on our surfaces some cross-shaped structures that resemble single molecules seen on mica ${ }^{37}$ can be sparsely observed on $\mathrm{X}_{\mathrm{OH}}=0.3$ and $\mathrm{X}_{\mathrm{OH}}=1$ surfaces (Fig. $3 \mathrm{~A}$, arrowheads), suggesting a protein deposition in a near-natural configuration.

\section{Cellular interaction with $L A M$}

Figure 4A describes the behavior of HUVEC on Lam adsorbed on the same substrates order. Again, two maxima in the cellular interaction were obtained at $\mathrm{X}_{\mathrm{OH}}=1$ and $\mathrm{X}_{\mathrm{OH}}=$ 0.3 surfaces. Overall, the cells look better spread in comparison to Col IV samples (Fig. 2), evident from the well-developed focal adhesion complexes (Fig. 4, column B) and prominent actin stress fibers (Fig. 4, column C). Likewise, the cellular interaction is abolished on $\mathrm{X}_{\mathrm{OH}}=0.7$ and $\mathrm{X}_{\mathrm{OH}}=0.5$ samples, which is confirmed from the quantitative measurements of both adhesion (Fig. 4D, left) and spreading (Fig. 4C, right), showing significantly lower values $(p<0.05)$. Interestingly, here both the adhesion and spreading to $\mathrm{X}_{\mathrm{OH}}=0.3$ and $\mathrm{X}_{\mathrm{OH}}=1$ override the values of control glass samples (Fig. 4D) although not significantly $(p>0.05)$. The overall morphology of cells on the control Lam-coated sample might be seen on Figure 5D. Again, a tendency for diminished cellular interaction on the most hydrophobic $\mathrm{X}_{\mathrm{OH}}=0$ surface was found (Fig. $4 \mathrm{~A}$ ).

This family of Lam-coated polymers was used for in vitro studies with other cell systems. While better adhesion of Schwann cells was obtained at $\mathrm{X}_{\mathrm{OH}}=0.8$ (close to the maximum $\mathrm{X}_{\mathrm{OH}}=0.7$ found with HUVEC), the best performance using neural progenitors was found for $\mathrm{X}_{\mathrm{OH}}=0.5$, on which HUVEC interact worst. ${ }^{40,41}$ Taken together, it points out the cell type specificity in the interaction with Lam-coated materials.

\section{Spontaneous interaction of HUVEC with bare substrata}

AFM images show areas of bare polymer, which raise an important question: do the cells adhere to the plain polymers 
FIG. 4. Overall morphology of HUVEC adhering to Lam-coated substrates with increasing $-\mathrm{OH}$ density (A). Bar $100 \mu \mathrm{m}$. Cells were stained also for vinculin (B) and actin (C) as in Figure 2. Bar $10 \mu \mathrm{m}$. Quantities for cell adhesion (D, left graph) and spreading (D, right graph) are compared to control glass samples (white bars).
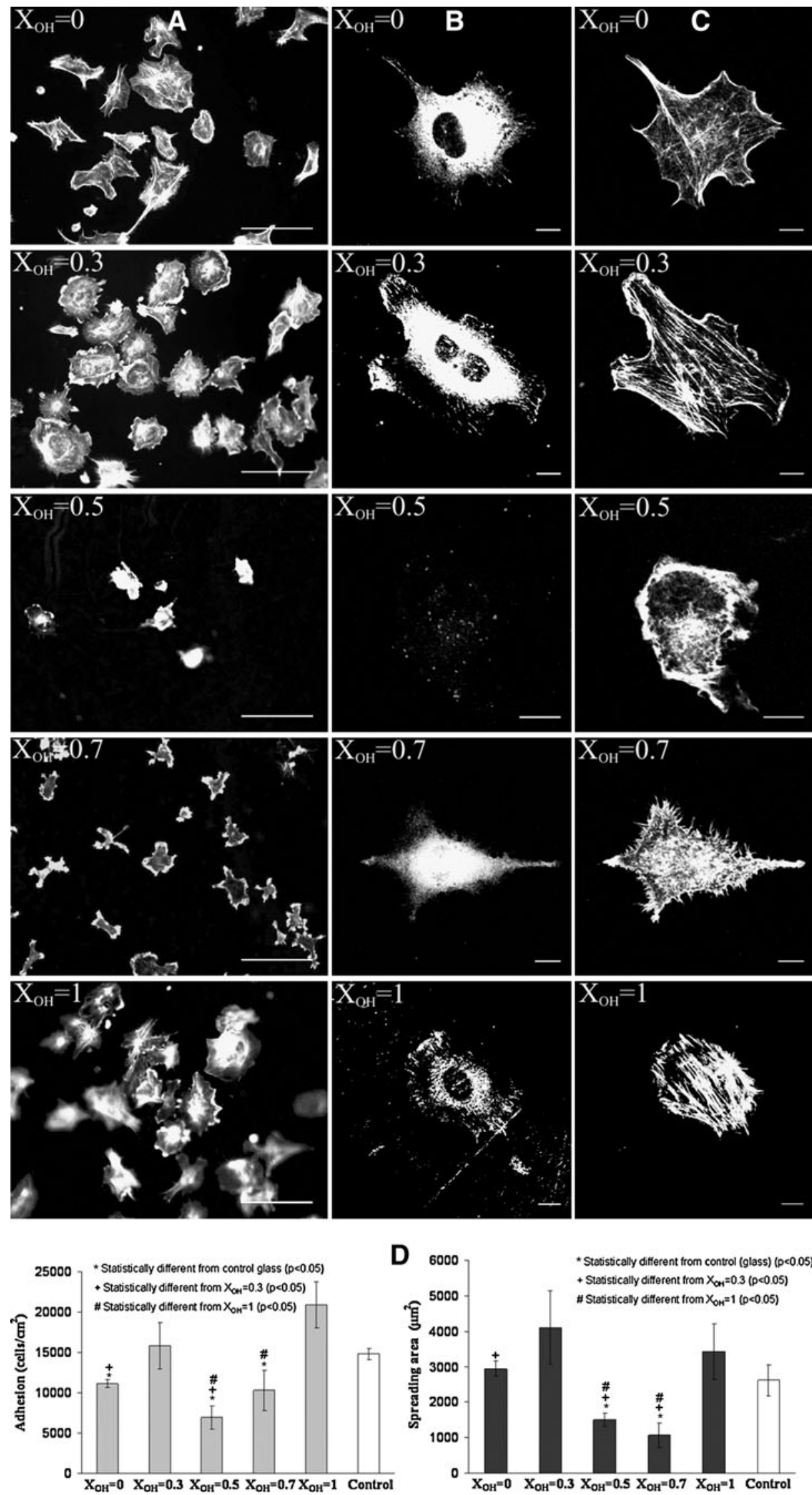
and does this adhesion vary with $-\mathrm{OH}$ density? To address this we performed separate experiment exploring the cellular interaction with bare surfaces. Figure 5 (column A) clearly demonstrates that the cells attach less efficiently and look shrink on all bare substrata suggesting a nonphysiological attachment, but a surprising tendency for higher attachment on $\mathrm{X}_{\mathrm{OH}}=1$ and $\mathrm{X}_{\mathrm{OH}}=0.3$ was found, further confirmed by the quantitative measures for cell adhesion $(p<0.05)$ (Fig. $5 \mathrm{H}$, upper chart), which suggests that these substrates provide better physical environment for cell attachment even in comparison to the control glass surface. However, it concerns cell adhesion, but not spreading, as the latter was even
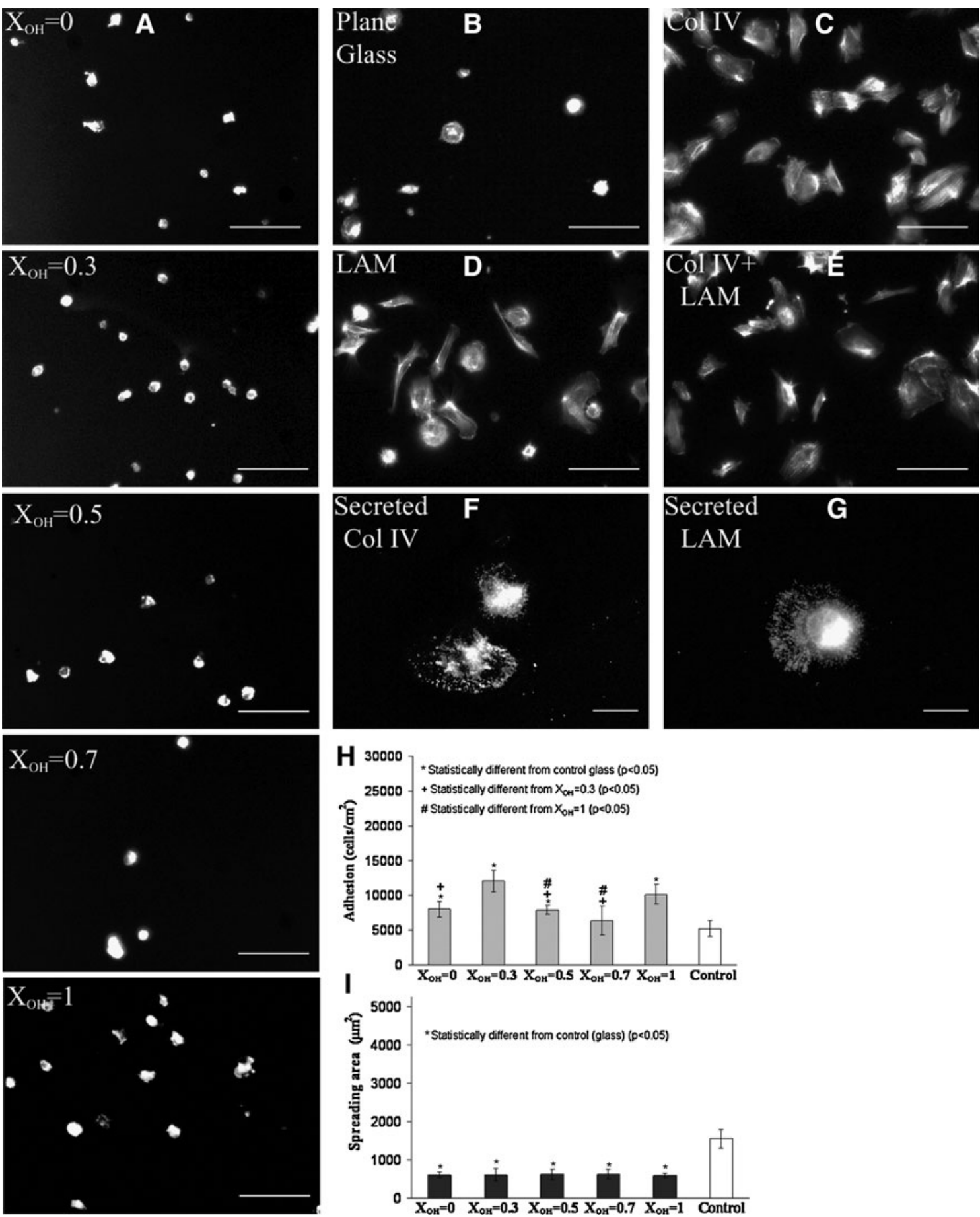

FIG. 5. Overall morphology of HUVEC adhering on plain substrates with increasing OH density (A), and different control samples including plain glass (B), Col IV-coated glass (C), Lam-coated glass (D), and sequential Col IV/Lam-coated glass (E). Bar $100 \mu \mathrm{m}$. The last two images demonstrate spontaneous secretion of Col IV (F) and Lam (G) by HUVEC within $2 \mathrm{~h}$ of incubation on glass. Bar $20 \mu \mathrm{m}$. The charts below represent quantities for cell adhesion (H) and spreading (I) to bare samples compared with glass control (white bars). 
lowered versus control glass (Fig. $5 \mathrm{H}$, lower chart), showing that these polymers provide conditions that are not sufficient to support the development of normal cell shape. On the other hand, it seemed unlikely that the cells adhere directly to bare substrates; therefore, the existence of some traces of adhesive proteins in the system might be expected. Presumably, they have to be secreted by HUVEC, as the effect of serum proteins can be excluded after the two times washing step we use for cell harvesting (See Methods section). Indeed, when HUVEC were stained for Col IV and Lam, both proteins were identified on the substratum beneath the cells even after $2 \mathrm{~h}$ incubation (Fig. $5 \mathrm{~F}$ and $\mathrm{G}$, respectively). Therefore, the improved spontaneous adhesion of HUVEC on $\mathrm{X}_{\mathrm{OH}}=1$ and $\mathrm{X}_{\mathrm{OH}}=0.3$ should be attributed to the constitutive release of Col IV and Lam (and may be other adhesive proteins), which influence the cellular interaction during the adhesion process. It is noteworthy, however, that this behavior of Col IV and Lam differs significantly from other matrix proteins, such as fibronectin ${ }^{17}$ and fibrinogen. ${ }^{19} \mathrm{Al}-$ though they also tend to assemble on pure PEA $\left(\mathrm{X}_{\mathrm{OH}}=0\right)$ the cellular interaction gradually increases as the fraction of $\mathrm{OH}$ groups diminishes. Conversely, vitronectin cannot form networks ${ }^{18}$ but show the same trend in bioactivity. The very similar biphasic behavior of HUVEC on Col IV and Lamcoated $\mathrm{X}_{\mathrm{OH}}=0.3$ and $\mathrm{X}_{\mathrm{OH}}=1$ substrata suggests that the adsorbed proteins acquire a configuration close to the natural one, and interestingly, single molecular-size features were observed for both proteins on $\mathrm{X}_{\mathrm{OH}}=0.3$ only (see arrow on Fig. 1A and arrowhead on Fig. 3A).

\section{Sequential deposition of Col IV and Lam}

The resemblances in the behavior of Col IV and Lam in respect to their assembly and cellular interaction suggest that similar forces might guide their organization in the BM, where they form overlapping polymeric network. ${ }^{10,13,24,42}$ This provoked our interest in a joint Col IV-Lam deposition, which could provide insights on their natural interplay in the BM. Unfortunately, these two proteins possess different solubility (Col IV dissolves in acid, whereas Lam in neutral conditions), which exclude their assembly together. Thus, the only simple solution was to adsorb them consequently, first Col IV and then Lam.

Figure 6A displays the complex phase images obtained after sequential Col IV/Lam adsorption. A tendency for joint network formation was found on most surfaces, except on $\mathrm{X}_{\mathrm{OH}}=1$, where rather globular protein deposition (resembling the behavior of Lam) was obtained (see Fig. 3, column A). It is still difficult to distinguish Col IV or Lam features, though clearly better network formation is seen on $\mathrm{X}_{\mathrm{OH}}=0.3$ sample. Sequential adsorption shows that the amount of adsorbed protein (see Table 2) is not just a superposition of these obtained for Col IV and Lam independently. Except for $\mathrm{X}_{\mathrm{OH}}=1$, it is always above these quantities (Table 2), which is consistent with the possibility that some Lam is additionally deposited on the surface. Interestingly, Lam tend to absorb preferentially on $\mathrm{X}_{\mathrm{OH}}=0.5$ and $\mathrm{X}_{\mathrm{OH}}=0.7$ surfaces after $\mathrm{Col}$ IV deposition than on the bare polymers (Table 2), suggesting heterotypic intermolecular interaction.

The structure of the protein layer looks different on PEA $\left(\mathrm{X}_{\mathrm{OH}}=0\right)$. Here prevails the homogenous "sponge-like" protein deposition interrupted by polygonal empty spaces,
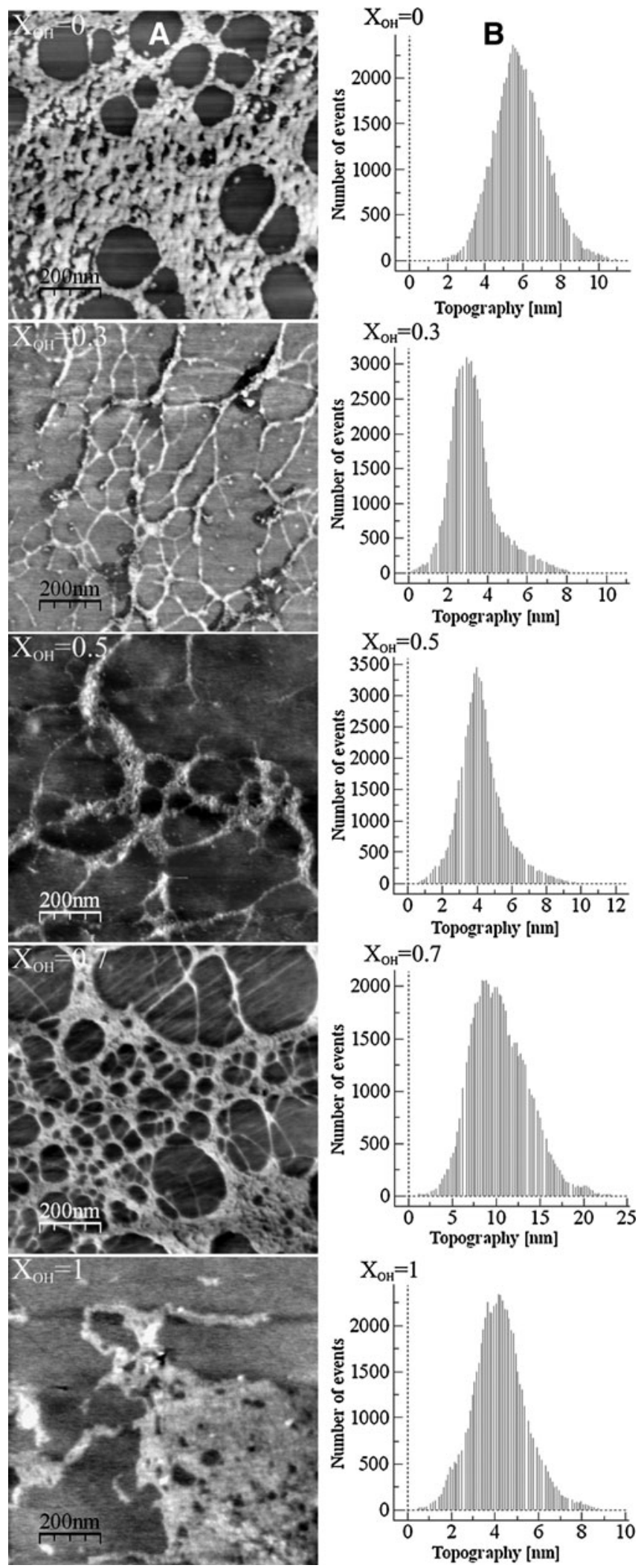

FIG. 6. Phase AFM images of sequentially adsorbed Col IV and Lam on substrates with increasing fraction of $-\mathrm{OH}$ groups (A) following the same order as in Figure 1. Corresponding topographic profiles of adsorbed proteins on the same substrates (B) viewed by AFM software. 

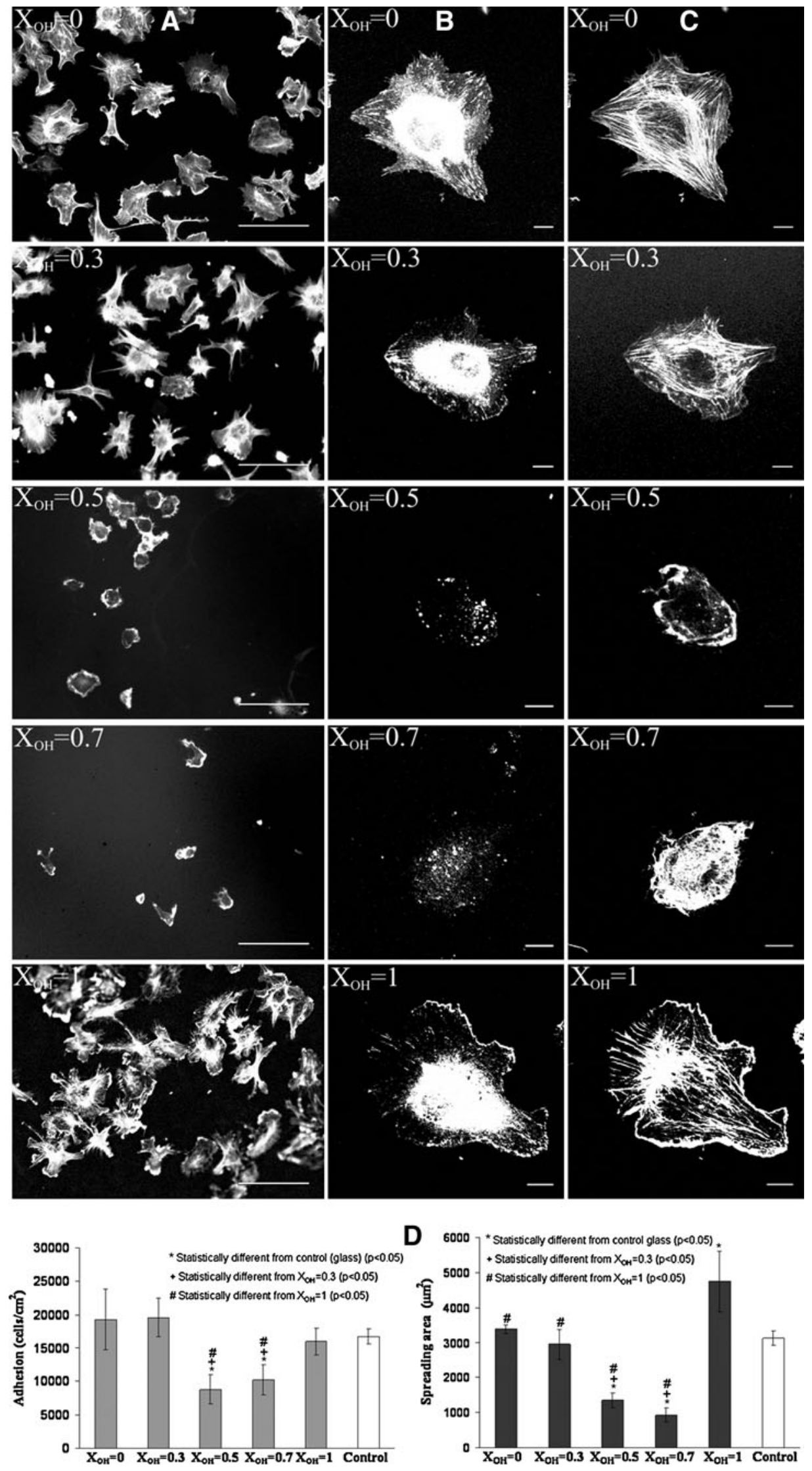

FIG. 7. Overall morphology of HUVEC adhering to sequentially adsorbed Col IV and Lam on substrates with increasing -OH density (A). Bar $100 \mu \mathrm{m}$. The cells were stained also for vinculin (B) and actin (C). Bar 10 $\mu \mathrm{m}$. Quantities for cell adhesion (D, left graph) and spreading (D, right graph) are compared to control glass samples (white bars). 
thus resembling the pattern of both Col IV (Fig. 1A) and Lam (Fig. 3A) networks. As Col IV adsorbs first it probably serves as a template for subsequent Lam assembly. The dominating sponge-like structure, however, presumably represents a joint Col IV-Lam network as it is not seen on the single protein series. Interestingly, the morphology of this complex network structure looks similar to those shown on the topographic AFM images of the natural BM underlying the corneal epithelium. ${ }^{43}$

The above assumption is confirmed by the improved cellular interaction that we obtained on $\mathrm{X}_{\mathrm{OH}}=0$ surface. As shown on Figure 7A, HUVEC represents the best morphology exactly on this sample demonstrating advanced cell spreading and polarized cell morphology reflecting their active crawling on the substratum. The most extensive formation of focal adhesions (Fig. 7B) and actin stress fibers (Fig. 7C) also confirm the improved cellular interaction. This suggests the appearance of a hydrophobic shift in bioactivity, confirmed also by the quantitative measurements, as cell spreading was significantly higher not only within the group, including control glass (Fig. 7D, right), but also compared to all other single protein series. Note, though the cells are well spread on $\mathrm{X}_{\mathrm{OH}}=0.3$ (optimum for single Col IV and Lam-coated series), they are often lacking their focal adhesions at cell borders, which points for a lowered strength of interaction with the substratum; that is, these samples start to represent lowered bioactivity when the matrix proteins are jointly assembled. Conversely, the complex Col-Lam network formation obviously supports the cellular interaction on the most hydrophobic $\mathrm{X}_{\mathrm{OH}}=0$ surface, which provides better environment for the cells presumably because it resembling the natural structure of the BM. ${ }^{43}$

\section{Conclusion}

This work describes the behavior of the main components of the BMs-Col IV and LAM-on well-defined surfaces with tailored density of hydroxyl groups. AFM studies revealed substratum-induced assembly of both proteins from a single-molecule arrangement to the specific networks better pronounced on hydrophobic environment.

The interaction of endothelial cells surprisingly shows two optima, a hydrophilic $\left(\mathrm{X}_{\mathrm{OH}}=1\right)$ one and a hydrophobic $\left(X_{\mathrm{OH}}=0.3\right)$ one, reveled from the changes in the adhering cells morphology, the quantities for cell adhesion and spreading, and the development of focal adhesion complexes. When both proteins were applied consequently, distinct complex morphology of the adsorbed protein layer was observed resulting in a "hydrophobic shift" in cellular interaction. In this article, we do not describe the specific conformation of individual molecules as it is difficult to follow with AFM on such rough environment. However, we show that the supramolecular assembly of both Col IV and Lam play an important role, which is the rationale of this work. Although at this time we cannot provide a real model for the functional interplay between proteins and cells, we could show the main players that affect the surface behavior of these matrix proteins: (1) the tendency (not obligatory!) for single molecule deposition on more hydrophilic substrata; (2) the hydrophobic environment provokes the assembly of proteins in networks (including joint Col IV-Lam networks); (3) these networks improve the cellular interaction (with optimum at $\mathrm{X}_{\mathrm{OH}}=0.3$ ), and (4) the very hydrophilic environment may also provoke the cellular interaction, presumably providing better molecular conformation (unfolded) of proteins that is more accessible for integrin receptors. Conversely, we do not state that these findings are universal, as our previous data show that other proteins like fibronectin, ${ }^{17,21}$ vitronectin, ${ }^{18}$ and fibrinogen ${ }^{19}$ behave differently on the same family of surfaces.

This work opens the door of using similar approaches to study different protein systems involved in cell-biomaterial interaction and BM organization.

\section{Acknowledgments}

AFM was performed under the technical guidance of the Microscopy Service at the Universidad Politécnica de Valencia, whose advice is greatly appreciated. The work was supported by the Spanish Ministry of Science and Innovation through project nos. MAT2009-14440-C02-0 and MAT200914440-C02-02.

\section{Disclosure Statement}

No competing financial interests exist.

\section{References}

1. Atala, A. Engineering organs. Curr Opin Biotechnol 20, 575, 2009.

2. Bueno, E.M., and Glowacki, J. Cell-free and cell-based approaches for bone regeneration. Nat Rev Rheumatol 5, 685, 2009.

3. Daley, W.P., Peters, S.B., and Larsen, M. Extracellular matrix dynamics in development and regenerative medicine. J Cell Sci 121, 255, 2008.

4. Griffith, L.G., and Naughton, G. Tissue engineeringcurrent challenges and expanding opportunities. Science (New York, N.Y.) 295, 1009, 2002.

5. Grinnell, F., and Feld, M.K. Fibronectin adsorption on hydrophilic and hydrophobic surfaces detected by antibody binding and analyzed during cell adhesion in serumcontaining medium. J Biol Chem 257, 4888, 1982.

6. Sipe, J.D. Tissue engineering and reparative medicine. Ann NY Acad Sci 961, 1, 2002.

7. de Mel, A., Jell, G., Stevens, M.M., and Seifalian, A.M. Biofunctionalization of biomaterials for accelerated in situ endothelialization: a review. Biomacromolecules 9, 2969, 2008.

8. Lutolf, M.P., and Hubbell, J.A. Synthetic biomaterials as instructive extracellular microenvironments for morphogenesis in tissue engineering. Nat Biotech 23, 47, 2005.

9. LeBleu, V.S., Macdonald, B., and Kalluri, R. Structure and function of basement membranes. Exp Biol Med (Maywood, N.J.) 232, 1121, 2007.

10. Timpl, R., and Brown, J.C. Supramolecular assembly of basement membranes. Bioessays News Rev Mol Cell Dev Biol 18, 123, 1996.

11. Rivron, N.C., Liu, J.J., Rouwkema, J., de Boer, J., and van Blitterswijk, C.A. Engineering vascularised tissues in vitro. Eur Cells Mater 15, 27, 2008.

12. Sephel, G.C., Kennedy, R., and Kudravi, S. Expression of capillary basement membrane components during sequential phases of wound angiogenesis. Matrix Biol 15, 263, 1996.

13. Van Agtmael, T., and Bruckner-Tuderman, L. Basement membranes and human disease. Cell Tissue Res 339, 167, 2010. 
14. Potenta, S., Zeisberg, E., and Kalluri, R. The role of endothelial-to-mesenchymal transition in cancer progression. $\mathrm{Br} \mathrm{J}$ Cancer 99, 1375, 2008.

15. Coelho, N.M., González-García, C., Planell, J.A., SalmerónSánchez, M., and Altankov, G. Different assembly of type IV collagen on hydrophilic and hydrophobic substrata alters endothelial cells interaction. Eur Cells Mater 19, 262, 2010.

16. Hernández, J.C.R., Salmerón Sánchez, M., Soria, J.M., Gómez Ribelles, J.L., and Monleón Pradas, M. Substrate chemistry-dependent conformations of single laminin molecules on polymer surfaces are revealed by the phase signal of atomic force microscopy. Biophys J 93, 202, 2007.

17. Gugutkov, D., Altankov, G., Rodriguez Hernandez, J.C., Monleon Pradas, M., and Salmeron Sanchez, M. Fibronectin activity on substrates with controlled-OH density. J Biomed Mater Res Part A 92, 322, 2009.

18. Toromanov, G., González-García, C., Altankov, G., and Salmerón-Sánchez, M. Vitronectin activity on polymer substrates with controlled -OH density. Polymer 51, 2329, 2010.

19. Hernández, J.C.R., Rico, P., Moratal, D., Pradas, M.M., and Salmerón-Sánchez, M. Fibrinogen patterns and activity on substrates with tailored hydroxy density. Macromol Biosci 9, 766, 2009.

20. González-García, C., Ferrus, L.L., Moratal, D., Pradas, M.M., and Sánchez, M.S. Poly(L-lactide) substrates with tailored surface chemistry by plasma copolymerisation of acrylic monomers. Plasma Processes Polym 6, 190, 2009.

21. Rico, P., Rodríguez Hernández, J.C., Moratal, D., Altankov, G., Monleón Pradas, M., and Salmerón-Sánchez, M. Substrate-induced assembly of fibronectin into networks: influence of surface chemistry and effect on osteoblast adhesion. Tissue Eng Part A 15, 3271, 2009.

22. Campillo-Fernández, A.J., Salmerón Sánchez, M., Sabater i Serra, R., Meseguer Dueñas, J.M., Monleón Pradas, M., and Gómez Ribelles, J.L. Water-induced (nano) organization in poly(ethyl acrylate-co-hydroxyethyl acrylate) networks. Eur Polym J 44, 1996, 2008.

23. Hudson, B.G., Reeders, S.T., and Tryggvason, K. Type IV collagen: structure, gene organization, and role in human diseases. Molecular basis of Goodpasture and Alport syndromes and diffuse leiomyomatosis. J Biol Chem 268, 26033, 1993.

24. Khoshnoodi, J., Pedchenko, V., and Hudson, B.G. Mammalian collagen IV. Microsc Res Tech 71, 357, 2008.

25. Fleischmajer, R., Perlish, J.S., Ii, D.E.M., Schechter, A., Murdoch, A.D., Iozzo, R.V., and Yamada, Y. There is binding of collagen IV to beta 1 integrin during early skin basement membrane assembly. Ann NY Acad Sci 857, 212, 1998.

26. Maneva-Radicheva, L., Ebert, U., Dimoudis, N., and Altankov, G. Fibroblast remodeling of adsorbed collagen type IV is altered in contact with cancer cells. Histol Histopathol 23, 833, 2008.

27. Timpl, R., Oberbaumer, I., von der Mark, H., Bode, W., Wick, G., Weber, S., and Engel, J. Structure and biology of the globular domain of basement membrane type IV collagen. Ann NY Acad Sci 460, 58, 1985.

28. Chen, C.H., and Hansma, H.G. Basement membrane macromolecules: insights from atomic force microscopy. J Struct Biol 131, 44, 2000.

29. Altankov, G., and Groth, T. Fibronectin matrix formation by human fibroblasts on surfaces varying in wettability. J Biomater Sci Polym Ed 8, 299, 1997.

30. Altankov, G., Groth, T., Krasteva, N., Albrecht, W., and Paul, D. Morphological evidence for a different fibronectin receptor organization and function during fibroblast adhesion on hydrophilic and hydrophobic glass substrata. J Biomater Sci Polym Ed 8, 721, 1997.

31. Kowalczynska, H.M., Nowak-Wyrzykowska, M., Kolos, R., Dobkowski, J., and Kaminski, J. Fibronectin adsorption and arrangement on copolymer surfaces and their significance in cell adhesion. J Biomed Mater Res Part A 72, 228, 2005.

32. Tamada, Y., and Ikada, Y. Fibroblast growth on polymer surfaces and biosynthesis of collagen. J Biomed Mater Res 28, 783, 1994.

33. Altankov, G., Grinnell, F., and Groth, T. Studies on the biocompatibility of materials: fibroblast reorganization of substratum-bound fibronectin on surfaces varying in wettability. J Biomed Mater Res 30, 385, 1996.

34. Beck, K., Hunter, I., and Engel, J. Structure and function of laminin: anatomy of a multidomain glycoprotein. FASEB J 4, 148, 1990.

35. Durbeej, M. Laminins. Cell Tissue Res 339, 259, 2010.

36. Tzu, J., and Marinkovich, M.P. Bridging structure with function: structural, regulatory, and developmental role of laminins. Int J Biochem Cell Biol 40, 199, 2008.

37. Chen, C.H., Clegg, D.O., and Hansma, H.G. Structures and dynamic motion of laminin-1 as observed by atomic force microscopy. Biochemistry 37, 8262, 1998.

38. Hansma, H.G. Varieties of imaging with scanning probe microscopes. Proc Nat Acad Sci USA 96, 14678, 1999.

39. Ortega-Vinuesa, J.L., Tengvall, P., and Lundstrom, I. Aggregation of HSA, IgG, and fibrinogen on methylated silicon surfaces. J Colloid Interface Sci 207, 228, 1998.

40. Liesi, P.I., Dahl, D., and Vaheri, A. Neurons cultured from developing rat brain attach and spread preferentially to laminin. J Neurosci Res 11, 241, 1984.

41. Soria, J.M., Ramos, C.M., Bahamonde, O., Cruz, D.M.G., Sánchez, M.S., Esparza, M.A.G., Casas, C., Guzmán, M., Navarro, X., Ribelles, J.L.G., Verdugo, J.M.G., Pradas, M.M., and Barcia, J.A. Influence of the substrate's hydrophilicity on the in vitro Schwann cells viability. J Biomed Mater Res Part A 83A, 463, 2007.

42. Charonis, A., Sideraki, V., Kaltezioti, V., Alberti, A., Vlahakos, D., Wu, K., and Tsilibary, E. Basement membrane peptides: functional considerations and biomedical applications in autoimmunity. Curr Med Chem 12, 1495, 2005.

43. Abrams, G.A., Goodman, S.L., Nealey, P.F., Franco, M., and Murphy, C.J. Nanoscale topography of the basement membrane underlying the corneal epithelium of the rhesus macaque. Cell Tissue Res 299, 39, 2000.

Address correspondence to: George Altankov, M.D., Ph.D. Institut de Bioenginyeria de Catalunya Feixa Llarga, s/n, Pavello Govern

$1^{\circ}$ Planta, Pta 1121 08907 L'Hospitalet de Llobregat

Barcelona Spain

E-mail: george.altankov@icrea.cat; altankov@abv.bg

Received: December 10, 2010 Accepted: May 03, 2011

Online Publication Date: June 16, 2011 
